\title{
Molecular beam epitaxy of GaBiAs on (311)B GaAs substrates
}

\author{
M. Heninia) \\ School of Physics and Astronomy, University of Nottingham, NG7 2RD Nottingham, United Kingdom \\ J. Ibáñez \\ Institut Jaume Almera, Consell Superior d'Investigacions Científiques (CSIC), 08028 Barcelona, Catalonia, \\ Spain
}

M. Schmidbauer

Institute for Crystal Growth, D-12489 Berlin, Germany

M. Shafi, S. V. Novikov, and L. Turyanska

School of Physics and Astronomy, University of Nottingham, NG7 2RD Nottingham, United Kingdom

S. I. Molina and D. L. Sales

Facultad de Ciencias, Universidad de Cádiz, 11510 Puerto Real, Cádiz, Spain

M. F. Chisholm

Materials Science and Technology Division, Oak Ridge National Laboratory, Oak Ridge, Tennessee 37831, USA

\author{
J. Misiewicz \\ Institute of Physics, Wroclaw University of Technology, 50-370 Wroclaw, Poland
}

(Received 15 October 2007; accepted 1 December 2007; published online 19 December 2007)

\begin{abstract}
We report the growth by molecular beam epitaxy of $\mathrm{GaBi}_{x} \mathrm{As}_{1-x}$ epilayers on (311)B GaAs substrates. We use high-resolution x-ray diffraction (HRXRD), transmission electron microscopy, and $Z$-contrast imaging to characterize the structural properties of the as-grown material. We find that the incorporation of $\mathrm{Bi}$ into the GaBiAs alloy, as determined by HRXRD, is sizably larger in the (311)B epilayers than in (001) epilayers, giving rise to reduced band-gap energies as obtained by optical transmission spectroscopy. (C) 2007 American Institute of Physics.
\end{abstract}

[DOI: $10.1063 / 1.2827181]$

$\mathrm{GaBi}_{x} \mathrm{As}_{1-x}$ alloys are attracting in recent years a considerable deal of attention. ${ }^{1-6}$ The band gap of GaAs is strongly reduced upon the addition of small amounts of $\mathrm{Bi}(\sim 90 \mathrm{meV}$ per percent of $\mathrm{Bi}$ ), with a strong enhancement of the spinorbit splitting energy. ${ }^{3,4}$ These remarkable properties make the $\mathrm{Ga}(\mathrm{In}) \mathrm{BiAs}$ system an attractive candidate to develop GaAs-based applications for long-wavelength optoelectronics as well as for spintronics. While the use of dilute nitrides in device applications is currently limited because the electron mobility of these compounds is abruptly reduced due to strong N-related alloy scattering, dilute bismides might exhibit improved transport properties. ${ }^{6}$

The growth of epitaxial layers on high-index planes represents a step forward in semiconductor material engineering, as it offers an additional degree of freedom to develop applications with improved properties with respect to the conventional (001)-grown devices. The interest in non(001) semiconductor structures is manifold and concerns growth, impurity incorporation, electronic properties, lasing performance, and piezoelectric effects. To mention a few examples, the use of non-(001) substrates has allowed the fabrication of ultrahigh mobility two-dimensional hole gases in GaAs/AlGaAs heterostructures, high-performance InAs/GaAs quantum dot (QD) lasers, InGaAs/GaAs QDs with enhanced piezoelectric effects, and GaMnAs epilayers with modified $\mathrm{Mn}$ incorporation and magnetic anisotropies. $^{7-9}$

\footnotetext{
${ }^{a)}$ Electronic mail: mohamed.henini@nottingham.ac.uk.
}

In this study, we explore the growth by molecular beam epitaxy (MBE) of GaBiAs alloys on (311)B GaAs substrates. We use a series of structural and optical techniques to characterize the as-grown material and to determine the amount of $\mathrm{Bi}$ incorporated into the samples. Our results reveal that the incorporation of $\mathrm{Bi}$ is enhanced in the (311) $B$ samples in comparison to the conventional (001) orientation.

For this study, $\mathrm{GaBi}_{x} \mathrm{As}_{1-x}$ films were grown by MBE on semi-insulating (001) and (311)B GaAs substrates at a growth temperature $\left(T_{G}\right)$ of $\sim 350^{\circ} \mathrm{C}$ with different As to $\mathrm{Ga}$ flux ratios. The growth was performed in a specially designed MBE reactor (see Ref. 10 for details). Atomic Ga and $\mathrm{Bi}$ were used as group-III and group-V sources, respectively, while As in the form of dimers $\left(\mathrm{As}_{2}\right)$ was produced by using a two-zone purpose made cell. In order to incorporate $\mathrm{Bi}$ efficiently into MBE-grown GaAs, the Ga to As flux needs to be on the brink of As shortage, close to the stoichiometric value. ${ }^{1,2}$ Thus, prior to the growth of the GaBiAs epilayers, we adjusted the Ga and As fluxes in order to deposit (001)and (311) $B$ GaAs under nominally stoichiometric conditions at both $\sim 350$ and $\sim 600^{\circ} \mathrm{C}$. Then, we fixed the Ga beamequivalent pressure (BEP) to a value of $\sim 9 \times 10^{-7}$ Torr for the growth of the (001) and (311)B GaBiAs epilayers $\left(T_{G}\right.$ $\left.\sim 350^{\circ} \mathrm{C}\right) . \mathrm{As}_{2} \mathrm{BEP}$ values were selected in the range from $6 \times 10^{-6}$ to $1.2 \times 10^{-5}$ Torr to achieve deposition under small excess of $\mathrm{Ga}$ or As. The Bi flux for the growth of GaBiAs was fixed at a BEP of $\sim 1.2 \times 10^{-7}$ Torr. Control GaAs samples without $\mathrm{Bi}$ were also grown on both (001) and (311) $B$ substrates at $T_{G} \sim 350$ and $\sim 600^{\circ} \mathrm{C}$. The nominal 


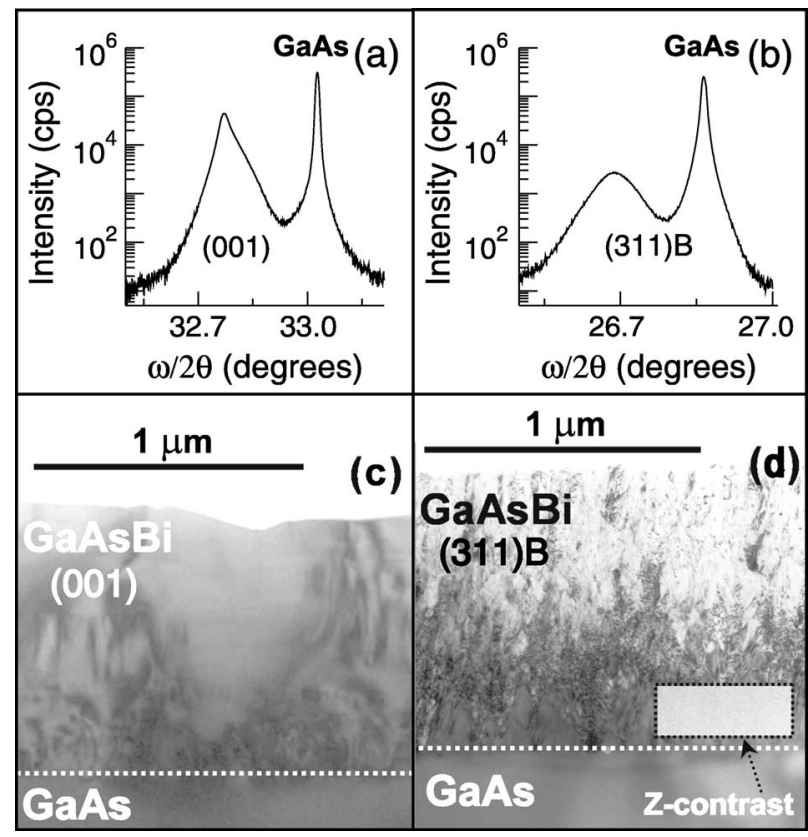

FIG. 1. HRXRD scans of two near-stoichiometric GaBiAs samples grown (a) on (001)-GaAs and (b) on (311)B GaAs. Bright field TEM images (c) of the (001) sample and (d) of the (311)B sample. Inset of (d): Z-contrast image of the (311) $B$ epilayer close to the GaBiAs/GaAs interface.

growth rate was around $1 \mu \mathrm{m} / \mathrm{h}$ for all the samples. The nominal thickness of all the epilayers is $1 \mu \mathrm{m}$. The specimens for transmission electron microscopy (TEM) and scanning transmission electron microscopy (STEM) were produced by mechanical thinning and ion milling. TEM images were taken at $120 \mathrm{kV}$ with a JEOL $1200 \mathrm{EX}$ microscope. STEM images were obtained at $300 \mathrm{kV}$ in a VG HB603U STEM microscope equipped with a Nion aberration corrector. Atomic number $(Z)$ contrast images were acquired with this instrument by using a high angle annular dark-field detector with an inner angle of $58 \mathrm{mrad}$. The samples were characterized with high-resolution x-ray diffraction (HRXRD) measurements. (004) and (311) GaAs symmetrical reflections were recorded for the (001) and the (311)B samples, respectively.

Figures 1(a) and 1(b) show selected HRXRD curves $(\omega / 2 \theta$ scans $)$ measured at an x-ray wavelength of $\lambda$ $=1.54 \AA\left(\mathrm{Cu} K \alpha_{1}\right)$ from two GaBiAs epilayers grown under near-stoichiometric conditions on (001) and on (311)B GaAs substrates. In addition to the reflections arising from the GaAs substrate, a broad diffraction peak located at lower diffraction angles is also observed in the HRXRD curves. This peak can be attributed to the GaBiAs alloy. ${ }^{1,2}$ Similar patterns were obtained for the rest of samples. It is interesting to note that, while the intensity of the substrate reflections is comparable for both (001) and (311)B samples [see Figs. 1(a) and 1(b)], the intensity of the layer reflections is much lower in the case of the (311) $B$ samples. This indicates that the (311) $B$ GaBiAs epilayers are appreciably thinner than the (001) epilayers, which suggests that the actual growth rate is greatly reduced for the (311) $B$ surface.

Asymmetric reciprocal space maps (not shown) revealed that, within experimental accuracy, the degree of plastic relaxation in all our samples is close to zero. Thus, the GaBiAs epilayers were grown coherently on both the (001) and the (311)B GaAs substrates. However, the width of the rocking Author complimentary copy. Redistribution subject to AIP

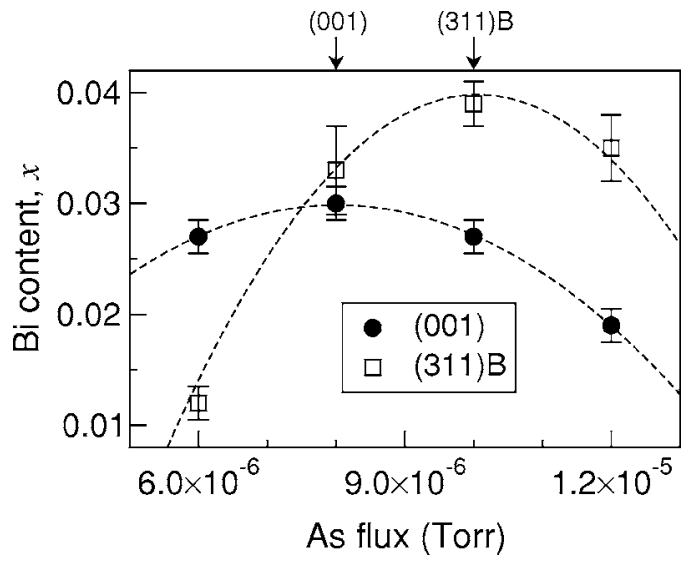

FIG. 2. Bi composition $x$ of different (001) and (311)B $\mathrm{GaBi}_{x} \mathrm{As}_{1-x}$ epilayers as a function of the As flux used for the growth. The arrows indicate the As flux for near-stoichiometric growth of (001) and (311)B GaAs.

curves, together with the fact that no interference fringes appeared in any of the HRXRD scans, suggests that a substantial degree of structural disorder and/or a highly inhomogeneous Bi distribution is present in these epilayers.

TEM images of the (001) and (311)B GaBiAs epilayers grown near stoichiometric conditions are shown in Figs. 1(c) and 1(d), respectively. These images reveal the presence of extended structural defects in both epilayers. In the case of the (001) GaBiAs film, such defects consist mainly of dislocations. In the case of the (311) $B$ sample, the TEM image shows that the crystalline quality of the GaBiAs epilayer is fairly good close to the GaBiAs/GaAs interface, where microtwins are observed. Closer to the free surface, however, the crystalline quality of the (311) $B$ epilayer degrades. The inset of Fig. 1(d) shows a $Z$-contrast image of this epilayer from an area close to the GaBiAs/GaAs interface. This image reveals that in spite of the extended disorder that is observed in the TEM images, no extended Bi segregation exists in these epilayers. Thus, we conclude that the observed broadening of the HRXRD peaks is mainly originated by the presence of extended defects in the samples.

The Bi concentration $x$ of the $\mathrm{GaBi}_{x} \mathrm{As}_{1-x}$ films can be determined from the Bragg angle of the GaAs and GaBiAs reflections as a function of the relaxed lattice parameters of $\mathrm{GaAs}$ and $\mathrm{GaBi}, a_{\mathrm{GaAs}}$ and $a_{\mathrm{GaBi}}$

$$
x=\left(\frac{a_{\mathrm{GaAs}}}{a_{\mathrm{GaBi}}-a_{\mathrm{GaAs}}}\right) P \cot \theta \Delta \theta,
$$

where $\theta$ is the Bragg angle for the GaAs substrate reflections and $\Delta \theta$ is the angle shift of the GaBiAs reflections with respect to the GaAs substrate. In the previous expression, $P$ represents the distortion coefficient, which depends on the elastic constants and on the surface orientation. ${ }^{11}$ Given that the $\mathrm{Bi}$ content of our samples is low, we use the elastic constants of GaAs to calculate $P$. We obtain $P_{100}=0.527$ and $P_{311}=0.691$ [see Ref. 11 for a detailed discussion about how to obtain $P$ in non-(001) films]. For the determination of $x$ we use the lattice parameter of the zincblende $\mathrm{GaBi}$ alloy estimated in Ref. 2, $a_{\mathrm{GaBi}}=6.33 \AA$. We plot in Fig. 2 the $\mathrm{Bi}$ content of all our (001) and (311) $B \mathrm{GaBi}_{x} \mathrm{As}_{1-x}$ epilayers, as obtained from the HRXRD scans. In the figure, $x$ has been plotted as a function of the As flux used to grow the films. The As flux required for near-stoichiometric growth for both the (001) and the (311)B samples is indicated with vertical license or copyright, see http://apl.aip.org/apl/copyright.jsp 


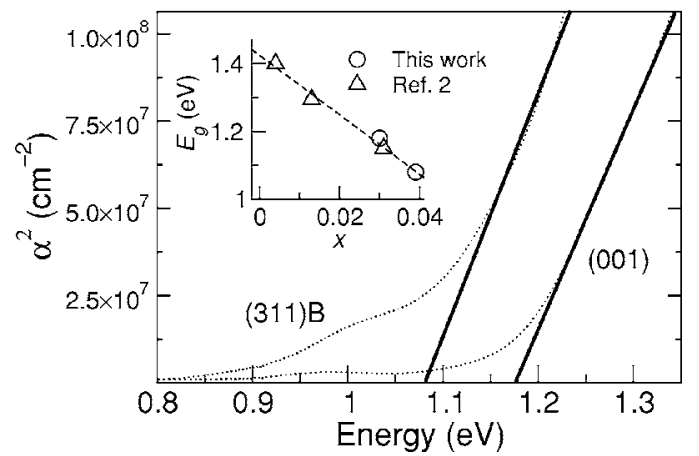

FIG. 3. Square of the absorption coefficient $\alpha^{2}$ for the two nearstoichiometric (001) and (311)B $\mathrm{GaBi}_{x} \mathrm{As}_{1-x}$ epilayers, obtained from transmission measurements at $300 \mathrm{~K}$. Inset: Band-gap energy $\left(E_{g}\right)$ vs $x$ for these two samples, together with data from Ref. 2.

arrows in the upper axis of the graph. The figure clearly shows that for both growth directions the maximum Bi composition is achieved near stoichiometric conditions, in agreement with previous reports on MBE-grown (001)-oriented GaBiAs. ${ }^{1,2}$ Remarkably, the Bi content incorporated into all the (311) $B$ epilayers, with the exception of the sample grown with the lowest As flux, is appreciably larger than that incorporated into the (001) samples. In the particular case of the (311) $B$ epilayer grown closest to stoichiometric conditions, we observe the largest $\mathrm{Bi}$ concentration $(x \sim 0.04)$.

The larger Bi content of the (311) $B$ epilayers gives rise to reduced band-gap energies $E_{g}$ with respect to the (001) samples. Figure 3 shows the square of the absorption coefficient $\alpha^{2}$ around the band-gap edge of the (001) and (311)B samples grown near stoichiometric conditions. These curves were obtained from conventional optical transmission measurements at $300 \mathrm{~K}$. For both samples, $\alpha^{2}$ displays an almost linear dependence on photon energy $E$, which is a consequence of the direct band gap of GaBiAs. By extrapolating the linear part of $\alpha^{2}(E)$ to zero, $E_{g}$ is obtained. Note that in Fig. 3 the $\alpha^{2}(E)$ curves have been obtained by taking the nominal thickness of the epilayers $(1 \mu \mathrm{m})$. However, as discussed above, the HRXRD data indicate that the (311) $B$ epilayer might be thinner. While an overestimation of the sample thickness would give rise to an underestimation of $\alpha^{2}$, this clearly does not affect the $E_{g}$ values obtained from the $\alpha^{2}(E)$ curves. We find that for the (311)B epilayer grown near stoichiometric conditions $E_{g}$ is around $90 \mathrm{meV}$ lower than for the corresponding (001) sample, as expected from the larger Bi content of the former $(x \sim 0.04$ versus $x \sim 0.03$, see Fig. 2). Preliminary photoluminescence (PL) measurements on these samples (not shown) corroborate these results. For comparison purposes, we have plotted in the inset of Fig. $3 E_{g}$ versus $x$ for the two stoichiometric samples, together with $E_{g}$ values of MBE-grown $\mathrm{GaBi}_{x} \mathrm{As}_{1-x}$ ( $x$ $<0.04)$ reported in Ref. 2. The plot shows that the band gap of the (311)B sample is consistent with the data from the (001) epilayers, thus, confirming the higher incorporation of Bi in the (311) $B$ surface.

Given that the incorporation of $\mathrm{N}$ into GaAsN alloys has been shown to decrease when the samples are grown on $B$-type (As-rich) non-(001) substrates, ${ }^{12}$ the larger Bi content of the (311) $B$ GaBiAs epilayers might seem surprising at a first sight. Note, however, that in $B$ surfaces the $\mathrm{Ga}$ atoms have two dangling bonds available, ${ }^{13}$ which suggests that the large $\mathrm{Bi}$ atom may be more easily incorporated at doubledangling bond sites while the small $\mathrm{N}$ atom may prefer the single-dangling bond sites. The differences in morphology, symmetry, and surface reconstruction may also be responsible for the different amounts of $\mathrm{Bi} / \mathrm{N}$ incorporated into the (001) and non-(001) surfaces.

Although the structural defects observed in our samples are not expected to affect strongly the incorporation of $\mathrm{Bi}$, their presence is clearly detrimental to the optical quality of the material. Here we speculate that the observed extended defects are generated after the growth of a critical thickness of nearly defect-free GaAsBi, as revealed in the TEM image of the (311)B epilayer [Fig. 1(d)]. Probably, after the growth of such critical thickness the defects propagate down to the interface to relax by plastic deformation. This is expected to complicate the subsequent evolution of the growth process, giving rise to polycrystalline grains and other types of defects. Accordingly, the higher density of defects observed in our stoichiometric (311) $B$ sample in the region closer to the surface could be a consequence of its higher Bi content (i.e., more strain to relax). While it is possible that the structural properties of our samples could be yet improved by further optimizing the growth conditions, we speculate that a much better structural quality would have been obtained with thinner epilayers.

In conclusion, increased $\mathrm{Bi}$ contents, leading to reduced band gaps, are achieved when GaBiAs is grown by MBE on (311) $B$ GaAs substrates in comparison to the conventional (001) orientation. The growth on (311)B surfaces of $\mathrm{Ga}(\mathrm{In})$ BiAs alloys has great potential for the development of GaAsbased long-wavelength device applications.

The work was supported by the UK EPSRC, the Spanish MEC (MAT2004-0664; TEC2005-05781-C03-02), SANDiE $\mathrm{NoE}$ and the Junta de Andalucia.

${ }^{1}$ K. Oe, Jpn. J. Appl. Phys., Part 1 41, 2801 (2002); M. Yoshimoto, S. Murata, A. Chayahara, Y. Horino, J. Saraie, and K. Oe, Jpn. J. Appl. Phys., Part 2 42, L1235 (2003).

${ }^{2}$ S. Tixier, M. Adamcyk, T. Tiedje, S. Francoeur, A. Mascarenhas, P. Wei, and F. Schiettekatte, Appl. Phys. Lett. 82, 2245 (2003).

${ }^{3}$ S. Francoeur, M. J. Seong, A. Mascarenhas, S. Tixier, M. Adamcyk, and T. Tiedje, Appl. Phys. Lett. 82, 3874 (2003); B. Fluegel, S. Francoeur, A. Mascarenhas, S. Tixier, E. C. Young, and T. Tiedje, Phys. Rev. Lett. 97, 067205 (2006).

${ }^{4}$ K. Alberi, O. D. Dubon, W. Walukiewicz, K. M. Yu, K. Bertulis, and A. Kroktus, Appl. Phys. Lett. 91, 051909 (2007).

${ }^{5}$ K. Bertulis, A. Kroktus, G. Aleksejenko, V. Pacebutas, R. Adomavicius, G. Molis, and S. Marcinkevicius, Appl. Phys. Lett. 88, 201112 (2006).

${ }^{6}$ D. G. Cooke, F. A. Hegmann, E. C. Young, and T. Tiedje, Appl. Phys. Lett. 89, 122103 (2006)

${ }^{7}$ M. Henini, P. J. Rodgers, P. A. Crump, B. L. Gallagher, and G. Hill, Appl. Phys. Lett. 65, 2054 (1994).

${ }^{8}$ A. Polimeni, M. Henini, A. Patanè, L. Eaves, P. C. Main, and G. Hill, Appl. Phys. Lett. 73, 1415 (1998); A. Patanè, A. Levin, A. Polimeni, F. Schindler, P. C. Main, L. Eaves, and M. Henini, ibid. 77, 2979 (2000).

${ }^{9}$ K. Y. Wang, K. W. Edmonds, L. X. Zhao, M. Sawicki, R. P. Campion, B. L. Gallagher, and C. T. Foxon, Phys. Rev. B 72, 115207 (2005).

${ }^{10}$ C. T. Foxon, I. Harrison, S. V. Novikov, A. J. Winser, R. P. Campion, T. Li, J. Phys.: Condens. Matter 14, 3383 (2002).

${ }^{11}$ J. Ibáñez, R. Kudrawiec, J. Misiewicz, M. Schmidbauer, M. Henini, and M. Hopkinson, J. Appl. Phys. 100, 093522 (2006).

${ }^{12}$ A. Arnoult, F. Gonzalez-Posada, S. Blanc, V. Bardinal, and C. Fontaine, Physica E (Amsterdam) 23, 352 (2004).

${ }^{13}$ B. Lee, S. S. Bose, M. H. Kim, A. D. Reed, G. E. Stillman, W. I. Wang, L. Viña, and P. C. Colter, J. Cryst. Growth 96, 27 (1989). 